Review Article

\title{
Chewing Gum for Intestinal Function Recovery after Colorectal Cancer Surgery: A Systematic Review and Meta-Analysis
}

\author{
Binbin Mei, ${ }^{1}$ Wenting Wang, ${ }^{1}$ Feifei Cui, ${ }^{2}$ Zunjia Wen, ${ }^{3}$ and Meifen Shen ${ }^{4}$ \\ ${ }^{1}$ Department of Neurosurgery, The First Affiliated Hospital of Soochow University \& School of Nursing Soochow University, \\ Suzhou 215006, China \\ ${ }^{2}$ Department of Intensive Care Unit, The First Affiliated Hospital of Soochow University, Suzhou 215006, China \\ ${ }^{3}$ Nanjing Children's Hospital, Nanjing 210000, China \\ ${ }^{4}$ Department of Neurosurgery, The First Affiliated Hospital of Soochow University, Suzhou 215006, China \\ Correspondence should be addressed to Meifen Shen; meifenshen@suda.edu.cn
}

Received 18 June 2017; Revised 21 August 2017; Accepted 6 September 2017; Published 8 October 2017

Academic Editor: Per Hellström

Copyright (C) 2017 Binbin Mei et al. This is an open access article distributed under the Creative Commons Attribution License, which permits unrestricted use, distribution, and reproduction in any medium, provided the original work is properly cited.

\begin{abstract}
Background. This meta-analysis was performed to assess the efficacy and safety of chewing gum in intestinal function recovery after colorectal cancer surgery. Methods. A systematic search was conducted in PubMed, Embase, Science Direct, and Cochrane library for relevant randomized controlled trials (RCTs) published until April 2017. Summary risk ratios or weighted mean differences with 95\% confidence intervals were used for continuous and dichotomous outcomes, respectively. Results. 17 RCTs with a total number of 1845 patients were included. Gum chewing following colorectal cancer surgery significantly reduced the time to first passage of flatus (WMD $-0.55 ; 95 \% \mathrm{CI}-0.94$ to -0.16 ; $P=0.006$ ), first bowel movement (WMD $-0.60 ; 95 \%$ CI -0.87 to -0.33 ; $P<0.0001$ ), start feeding (WMD $-1.32 ; 95 \%$ CI -2.18 to $-0.46 ; P=0.003$ ), and the length of postoperative hospital stay (WMD $-0.88 ; 95 \%$ CI -1.59 to $-0.17 ; P=0.01$ ), but no obvious differences were found in postoperative nausea, vomiting, abdominal distention, pneumonia, and mortality, which were consistent with the findings of intention to treat analysis. Conclusions. Chewing gum could accelerate the recovery of intestinal function after colorectal cancer surgery. However, it confers no advantage in postoperative clinical complications. Further large-scale and high-quality RCTs should be conducted to confirm these results.
\end{abstract}

\section{Introduction}

Colorectal cancer is one of the most common tumors, which is associated with multiple risk factors and accounts for approximately $10 \%$ of cancer-related mortality in western countries $[1,2]$. With increased colorectal cancer incidence, the number of colorectal cancer surgery has also elevated dramatically. Laparoscopic or open colorectal resections, as the main surgical approach for colorectal cancer, may also result in many serious complications like postoperative ileus, nausea, and vomiting, which could lead to significant postoperative morbidity and a prolonged length of hospital stay and place a significant financial burden on health care facilities $[3,4]$. Therefore, targeting the recovery of intestinal function after colorectal cancer surgery may contribute to promote rehabilitation for the patient. Now, it is widely accepted that early postoperative feeding is beneficial to decrease the postoperative ileus and shorten the length of hospital stay, and there is significant interest in identifying the measures to stimulate gut function, rather than simply waiting for it to return spontaneously [5-7].

Gum chewing (GC), a kind of sham feeding alternative to early feeding, which is expected to stimulate the cephalicvagal reflex to increase hormone secretion and then enhance intestinal motility [8], may produce a positive effect on postoperative ileus by reducing postoperative inflammation [9]. However, the underlying mechanisms behind the effects of gum chewing works remain elusive and the results of clinical 
studies to date are inconclusive, and thus chewing gum has not yet been incorporated into the related guidelines or standard pathways for postoperative nursing $[10,11]$.

Previous meta-analyses [7, 12-14] have suggested that patients who had gum chewing after surgery might recover intestinal function earlier; however, the sample sizes of the randomized controlled trials (RCTs) included in these analyses were small and an increasing body of relevant research recently have been reported, making it worth reconsidering the evidence on this issue. The aims of this study, therefore, were to review the current evidence on the influence of gum chewing in intestinal function and reassess the efficacy of chewing gum in intestinal function recovery after colorectal surgery.

\section{Methods}

2.1. Search Strategy. A general literature search was conducted in PubMed, Embase, Science Direct, and Cochrane library (until April 2017), using the following search terms: "gum-chewing," "chewing-gum," "sham-feeding," "bowel," "colonic," "rectal," "resection," or "surgery." We combined these terms in accordance with the instructions of the database. Reference lists and bibliographies from included studies and relevant reviews were used to find additional articles to review. The search was limited to articles written in English, but there was no restriction on publication year.

2.2. Study Selection. Two reviewers independently reviewed titles and abstracts to distinguish potentially relevant studies, and articles for more extensive review were selected using the following criteria. Inclusion criteria were as follows: (1) RCT design; (2) study subjects were those who had undergone colorectal cancer surgery; (3) the study made a comparison between gum chewing and standard nursing care post colorectal surgery; and (4) the study contained explicit outcome data. Exclusion criteria were as follows: (1) reviews, systematic reviews, meta-analysis, editorials, case studies, and nonhuman studies; (2) studies not focusing on the effect of chewing gum on colorectal cancer surgery; and (3) studies with no accessible outcome data.

The main outcomes we collected for data analysis included (1) the time to first passage of flatus (days); (2) the time to first defecation (days); (3) the time to first bowel movement (days); (4) the time to start feeding (days); (5) postoperative ileus (POI); (6) postoperative clinical complications such as nausea, vomiting, abdominal distension, and pneumonia; (7) the length of postoperative hospital stay (days); and (8) mortality.

2.3. Quality Assessment. The methodological quality and risk of bias of included studies were assessed using the Cochrane Collaboration's "risk of bias" tool, which addresses the seven specific domains: random sequence generation, allocation concealment, blinding of participants and personnel, blinding of outcome assessment, incomplete outcome data, selective reporting, and other sources of bias. Each domain in the tool is achieved by assigning a judgement of "low risk" of bias, "high risk" of bias, or "unclear risk" of bias
(Cochrane Handbook for Systematic Reviews of Intervention. Part 2: 8.5) [15].

2.4. Data Extraction. Two reviewers independently extracted the following data information: first author, publication year, study design, participants, population, methods of gum chewing, other concurrent interventions, main outcomes, and finding. Disagreements were resolved by discussion or, when necessary, adjudicated by a third reviewer.

2.5. Statistical Analysis. Review Manager 5.3 software was used for statistical analyses. We calculated risk ratio (RR) and weighted mean difference (WMD) to present the dichotomous and continuous data, respectively, and 95\% confidence interval (CI) was included for all estimates. "Intention to treat" analysis was also conducted. $P<0.05$ indicated statistical significance. Heterogeneity was tested by applying a chi-squared $\left(\chi^{2}\right)$ test and I-squared $\left(I^{2}\right)$ statistic test. A fixed-effect model was adopted when $P$ value of $\chi^{2}$ test $>0.10$ and $I^{2} \leq 50 \%$, and a random-effect model was adopted otherwise.

\section{Results}

3.1. Literature Search. Using the search strategy, 295 potentially relevant references were identified. Of these, 278 articles were excluded due to duplicates or their failure to fulfil predefined inclusion criteria. So finally, we formally included 17 trials with 1845 patients in the main analyses of this review [16-32] (Figure 1).

3.2. Study Characteristics. The characteristics of 17 included studies are presented in Table 1 . The included studies comprised a total of 1845 patients who had received surgical treatment for colorectal cancer. Of these, 918 followed a gum chewing intervention, and 927 received standard postoperative care. For most studies, gum chewing started from the first day post operation, and the frequency varied from three to four times per day. Although a lot of research confirmed the benefits of chewing gum, the outcomes for the efficacy of gum chewing in each study were still controversial.

3.3. Methodological Quality and Risk of Bias. Figure 2(a) describes all of the bias classifications for the included RCTs, with the summary of qualitative methodological quality according to the bias classification ("low risk," "unclear risk," and "high risk") which was presented in Figure 2(b). Concisely, although all included RCTs mentioned randomization in their research, only nine studies $[17,19,20,24,25,27,29,31,32]$ reported correct method of random sequence generation which marked as low risk of selection bias, and only six RCTs $[17,20,24,25,27,31]$ described adequate allocation concealments, which serves as a strategy to prevent against ascertainment bias and scored as low risk of bias. In general, sequentially numbered, opaque, sealed envelopes are assigned to each participant to prevent selective bias. Adequate blinding of personnel, participants, and outcome assessment is necessary to prevent against performance and detection bias; however, only one study [25] explicitly described how to maintain patient blinding in all included research, and six RCTs $[17,24,25,27,29,31]$ had mentioned 


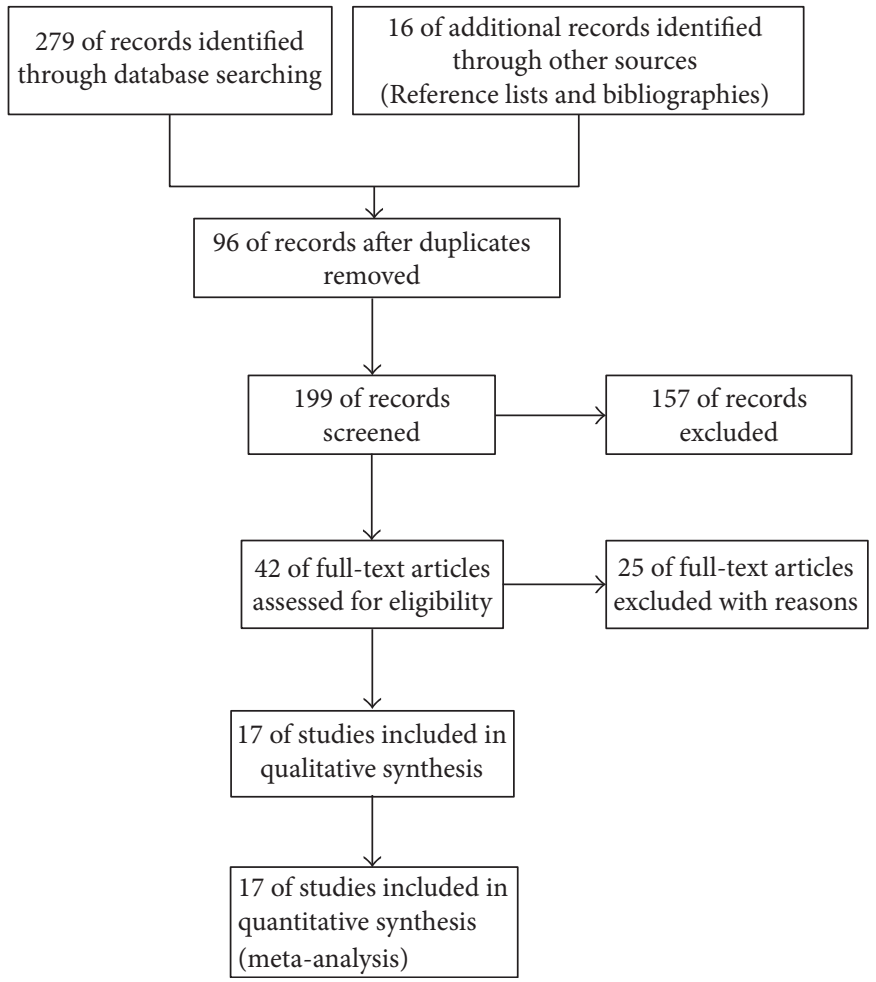

FIgURE 1: Study flow diagram.

the blinding design for assessing the outcomes. Verification on selective reporting of outcomes is necessary because it may help to evaluate the integrity of outcome reporting and protect against bias, yet the majority of the included studies [16, 18-23, 25-29, 31, 32] did not provide sufficient information to assess whether an important risk of bias exists; thus, we judged the risk of other potential sources of bias as unclear. Most of the included studies appear to be free of other sources of bias except for two studies $[26,32]$, so we judged the risk of bias as low risk for these trials.

3.4. Publication Bias Analysis. A funnel plot is a simple scatter plot of the intervention effect estimates from individual studies against some measure of each study's size or precision which is used for indication of publication bias [15]. Ten or more studies are needed to enable a funnel plot to give significant evidence of bias, and in this study, we performed a funnel plot analysis on the time to first passage of flatus and the length of postoperative hospital stay. Based on Figures 3(a) and 3(b), we found that the funnel plots were symmetrical, and no significant publication bias was found.

3.5. Main Analysis. The effect of gum chewing on multiple parameters of colorectal function following surgery reported in the included studies was assessed. The RRs or WMDs for each study were presented in Figures 4-14.

3.5.1. The Time to First Passage of Flatus. All studies reported the time to the first passage of flatus, yet seven studies $[17,18,20,22,24,29,31]$ failed to provide accurate and necessary data for further analysis. As shown in Figure 4, we found that gum chewing probably shorten the time to first passage of flatus (WMD $-0.55 ; 95 \%$ CI -0.94 to $-0.16 ; P=0.006)$ with a clear statistical heterogeneity across the trials $\left(I^{2}=78 \%\right)$.

3.5.2. The Time to First Defecation. Four studies reported the time to first defecation after colorectal surgery under gum chewing intervention or standard nursing. We found no evidence of a statistically significant difference regarding the time to first defecation between the gum chewing group and control group (WMD -0.96 ; 95\% CI -2.74 to $0.83 ; P=0.29$ ) (Figure 5 ), with a clear statistical heterogeneity between trials $\left(I^{2}=88 \%\right)$.

3.5.3. The Time to First Bowel Movement. Six studies reported the time to first bowel movement; we found that gum chewing shorten the time to first bowel movement compared with the control group (WMD -0.60 ; 95\% CI -0.87 to $-0.33 ; P<0.0001$ ) (Figure 6 ), with a moderate statistical heterogeneity across the studies $\left(I^{2}=41 \%\right)$.

3.5.4. Time to Start Feeding. Only two studies reported the time to start feeding; the meta-analysis showed a statistically significant reduction in the time to start feeding for the gum chewing group (WMD -1.32 ; $95 \% \mathrm{CI}-2.18$ to -0.46 ; $P=0.003$ ) (Figure 7), with no evidence of heterogeneity across the trials ( $P$ value of the homogeneity test $=0.79$; $\left.I^{2}=0 \%\right)$.

3.5.5. Postoperative Ileus (POI). No significant difference was observed between the gum chewing group and control group 


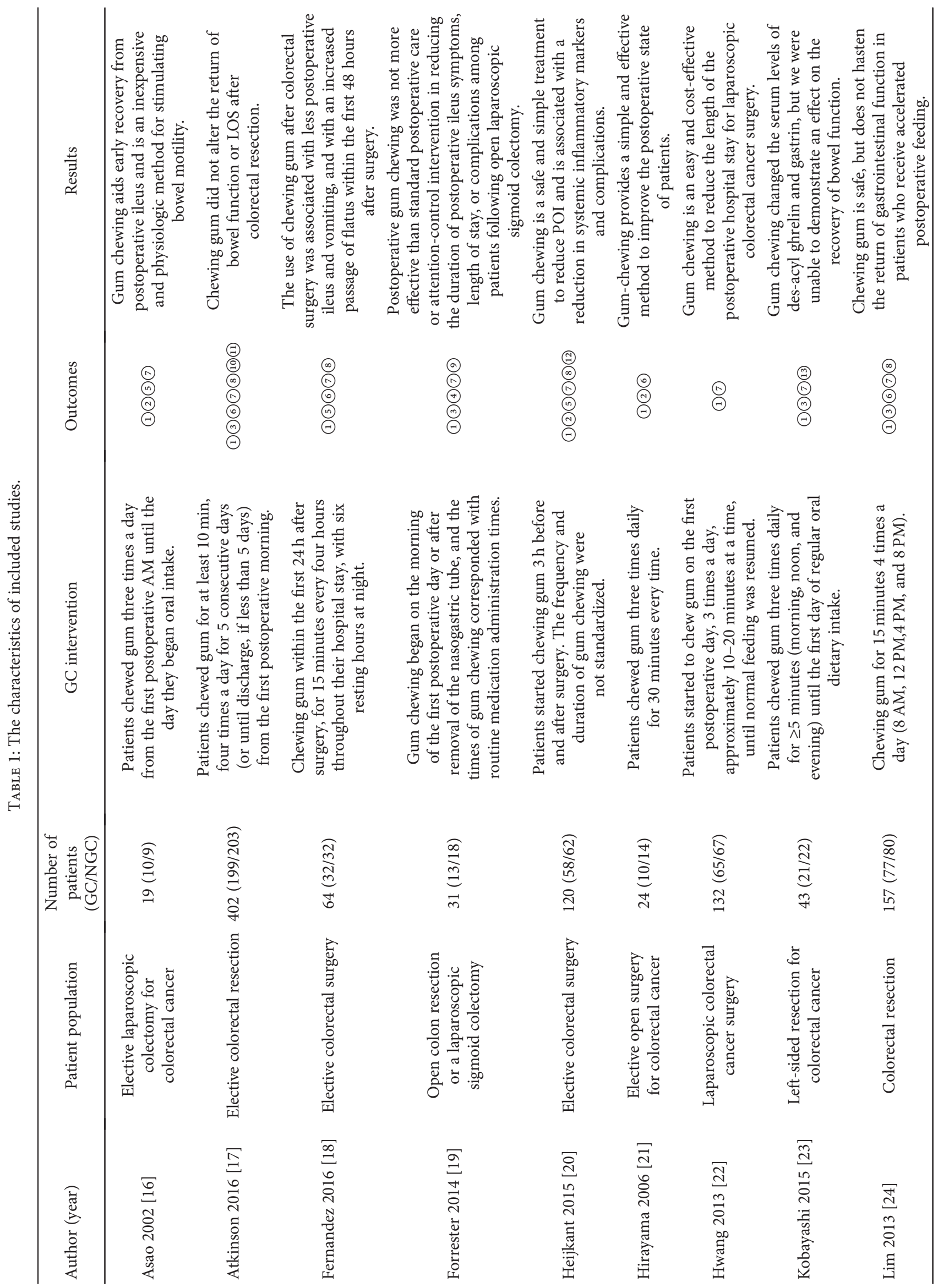




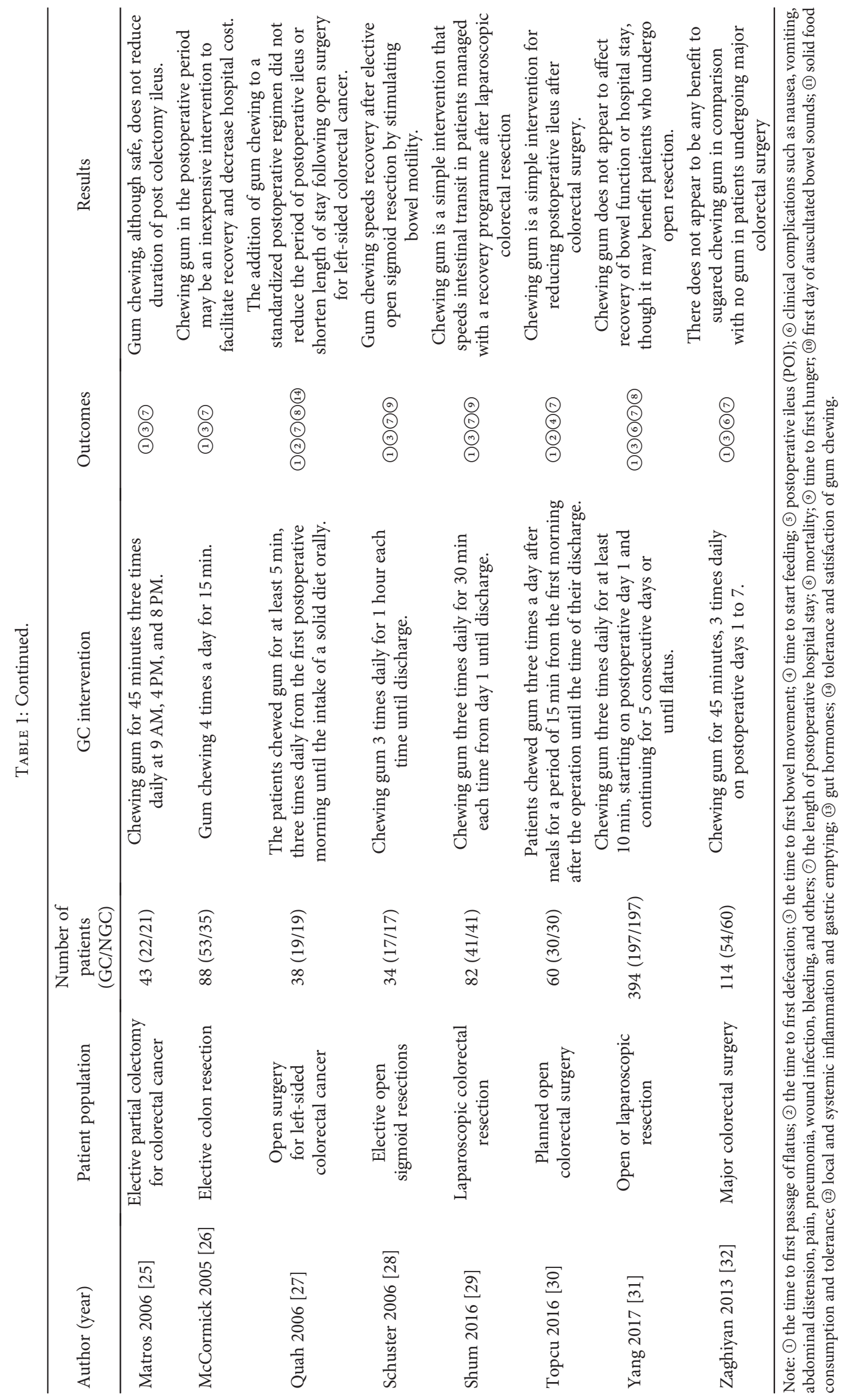




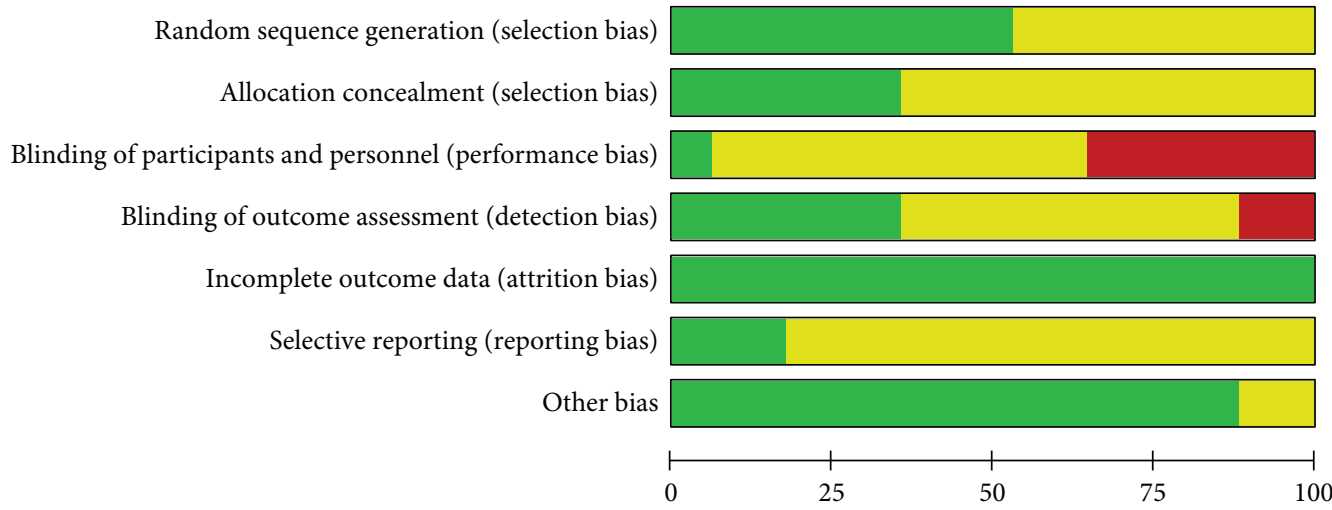

(\%)

Low risk of bias

Unclear risk of bias

High risk of bias

(a) Risk of bias graph

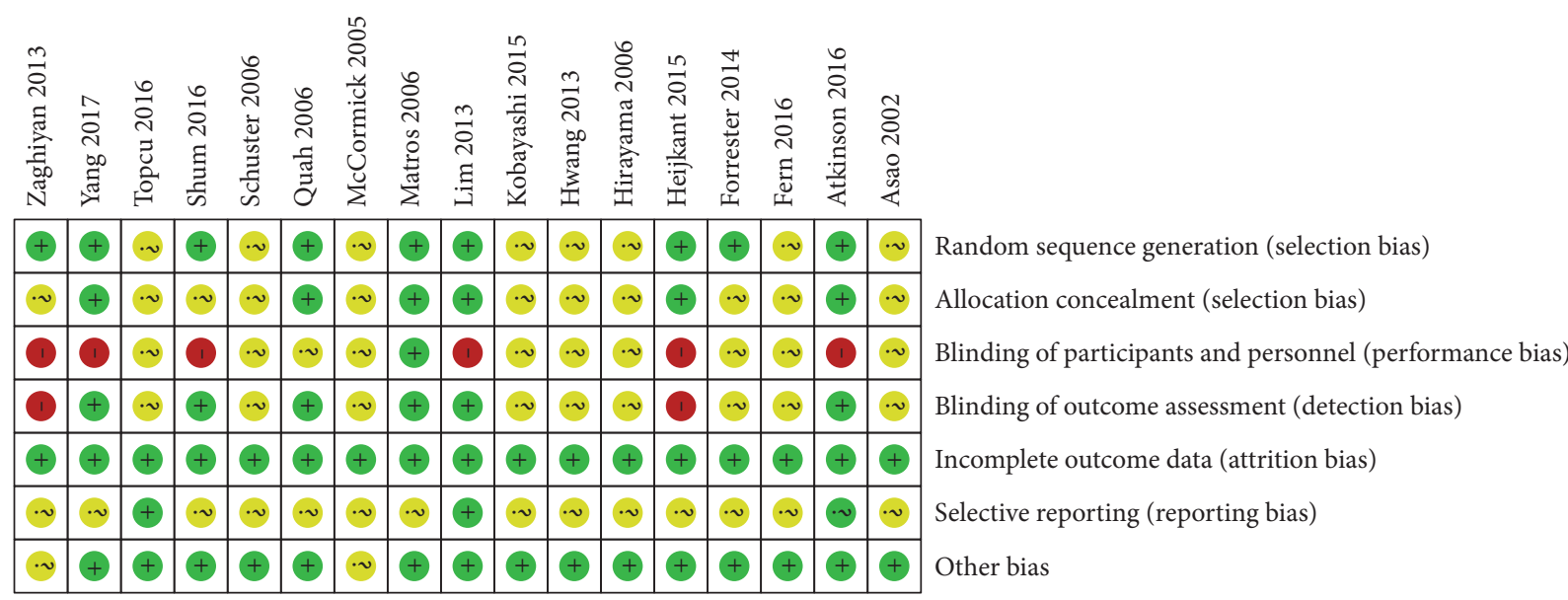

(b) Risk of bias summary

Figure 2: Methodological quality and risk of the included studies.

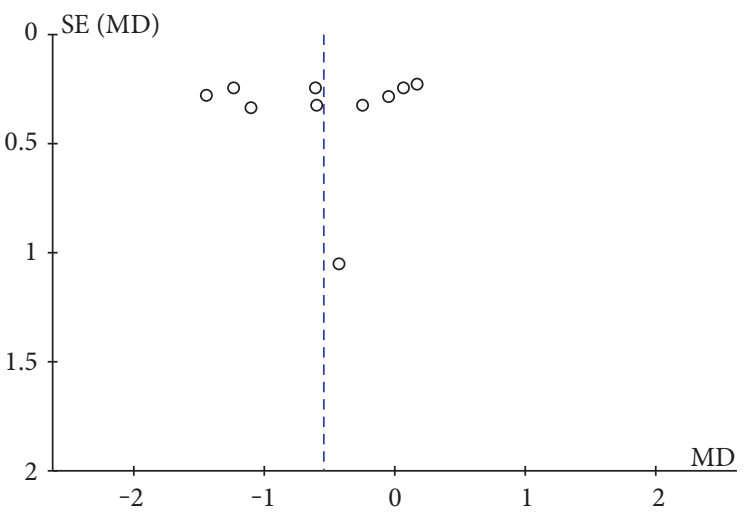

(a) Funnel plot for the time to first passage of flatus

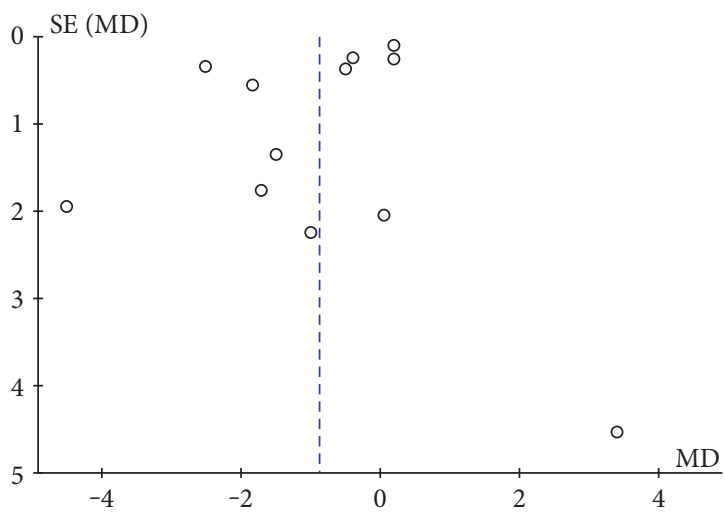

(b) Funnel plot for the length of postoperative hospital stay

Figure 3

in the incidence of POI (RR 0.29; 95\% CI 0.08 to 1.11 ; $P=0.07)$ (Figure 8), with no statistical heterogeneity between trials $\left(P=0.97 ; I^{2}=0 \%\right)$.
3.5.6. Postoperative Nausea. There was no significant difference between the groups in the incidence of postoperative nausea (RR $0.97 ; 95 \%$ CI 0.82 to $1.15 ; P=0.74$ ) 


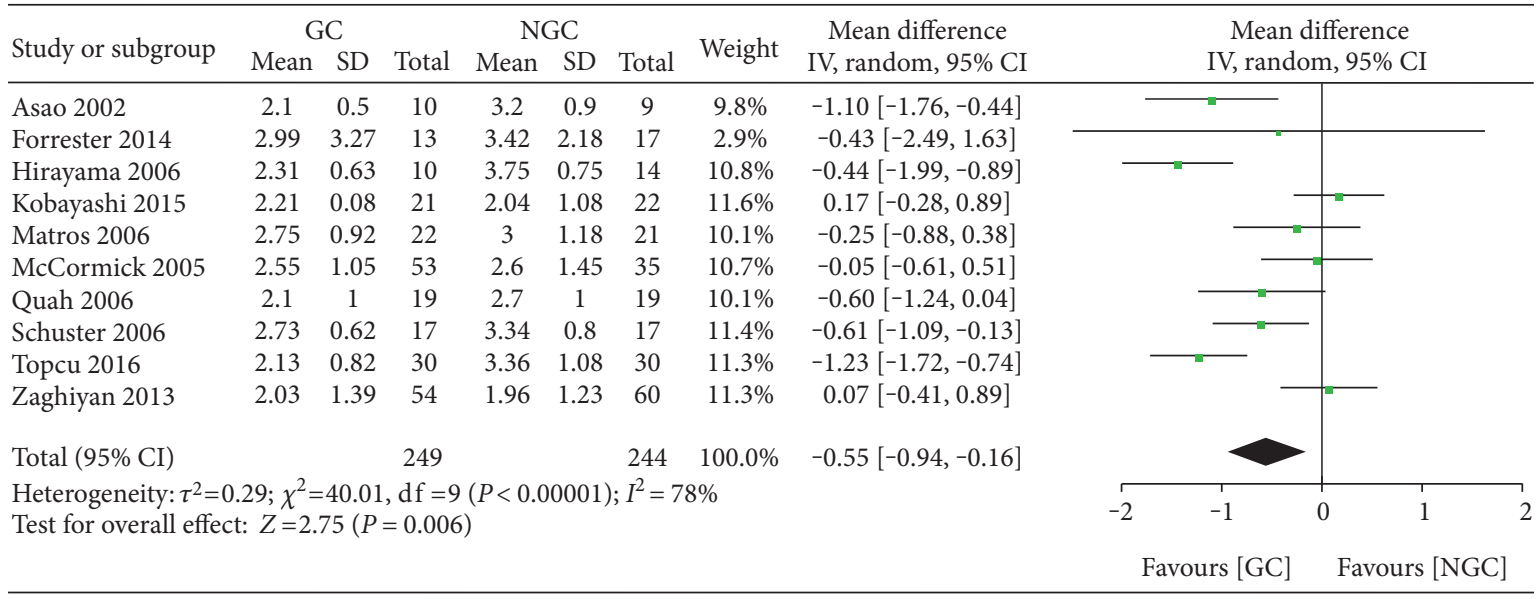

FIGURE 4: Forest plot for the time to first passage of flatus.

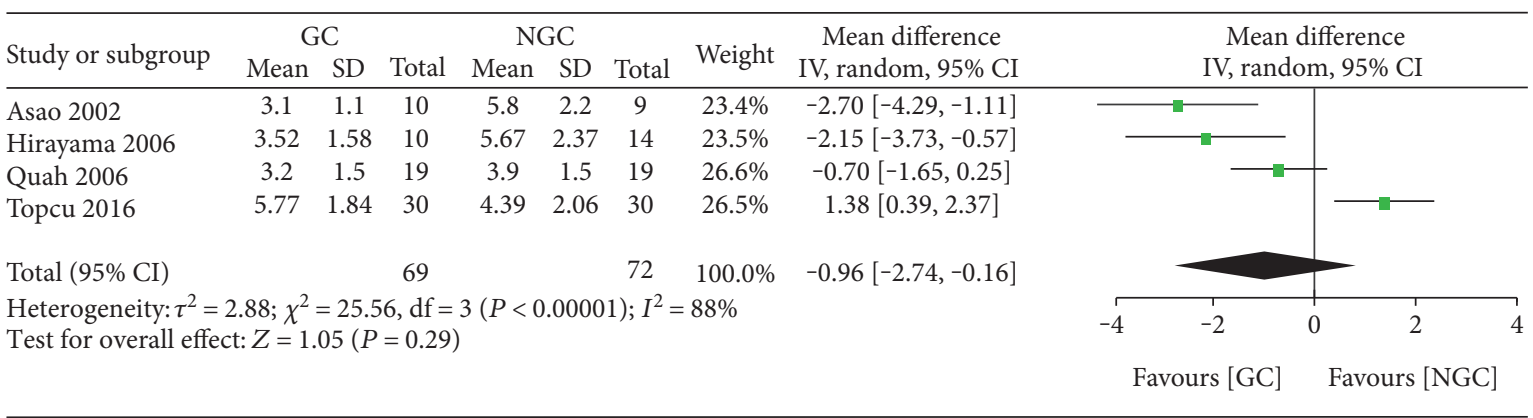

Figure 5: Forest plot for the time to first defecation.

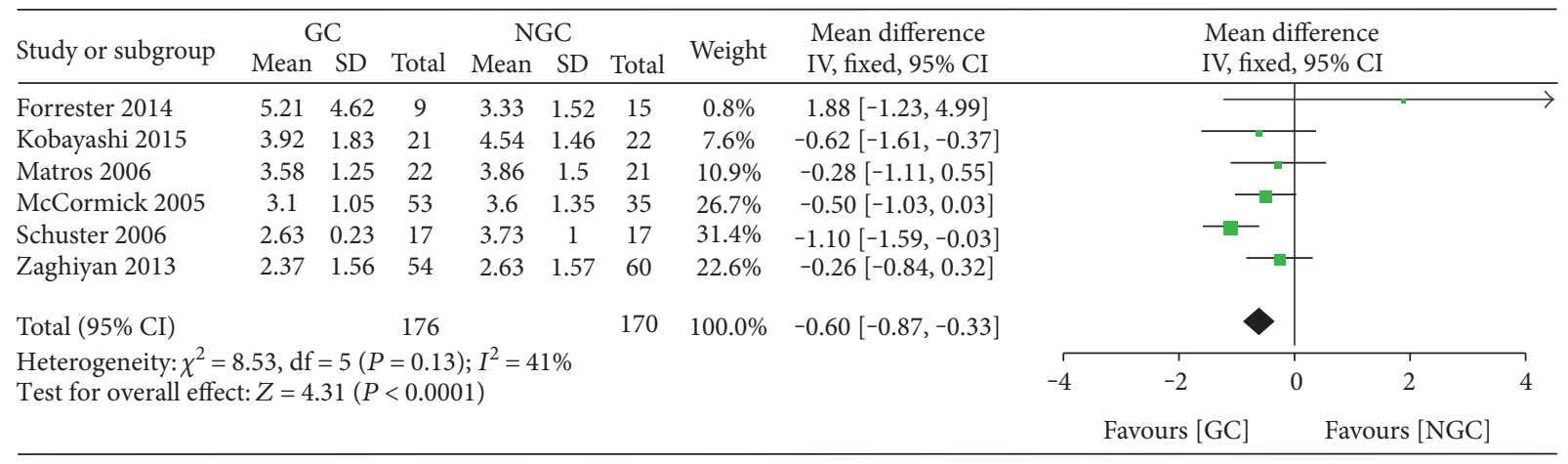

FIgURE 6: Forest plot for the time to first bowel movement.

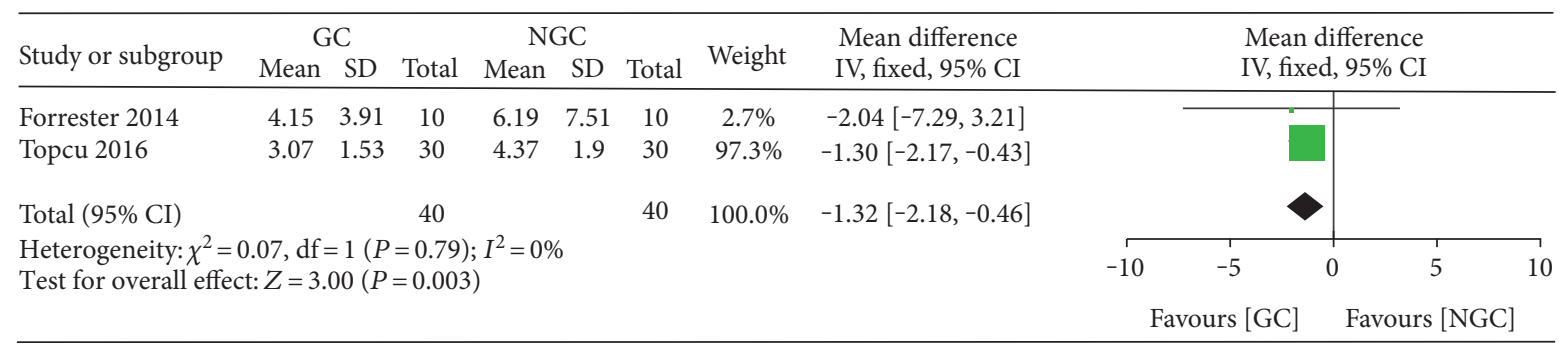

FIGURE 7: Forest plot for the time to start feeding. 


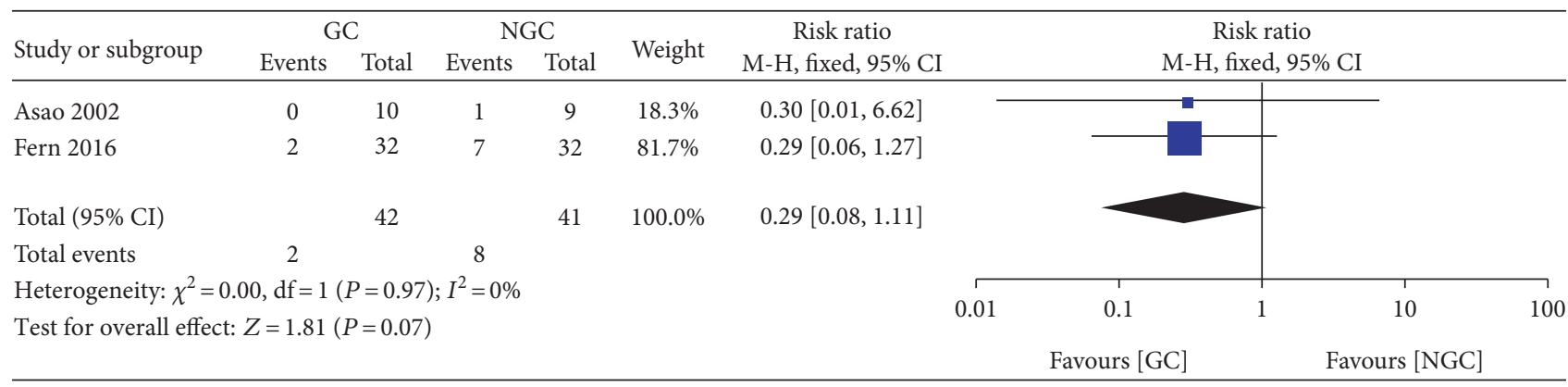

FIgURE 8: Forest plot for postoperative ileus.

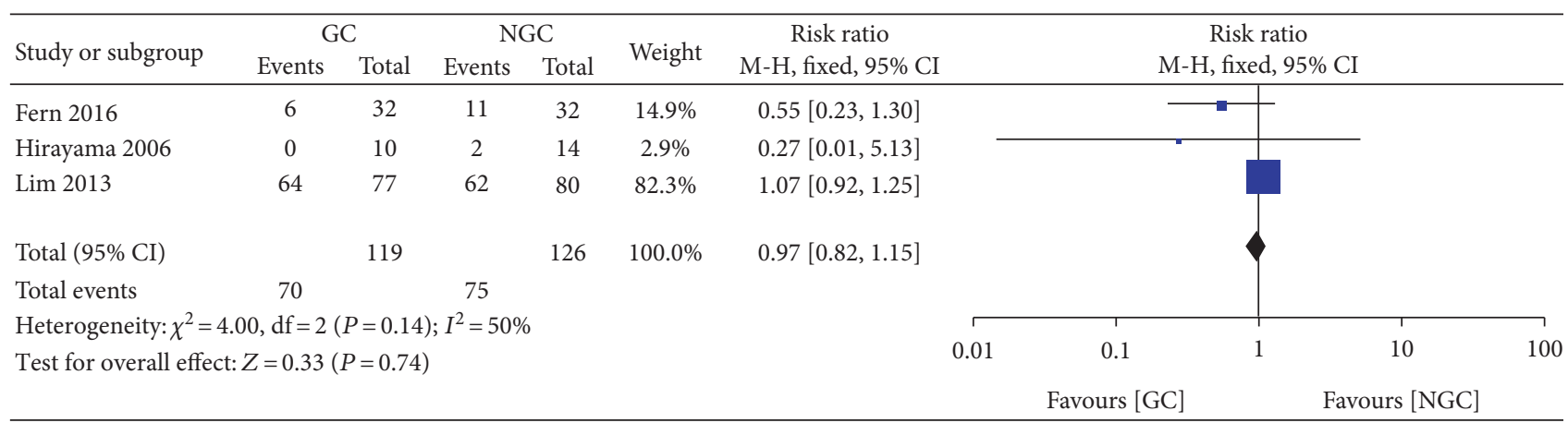

FIGURE 9: Forest plot for postoperative nausea.

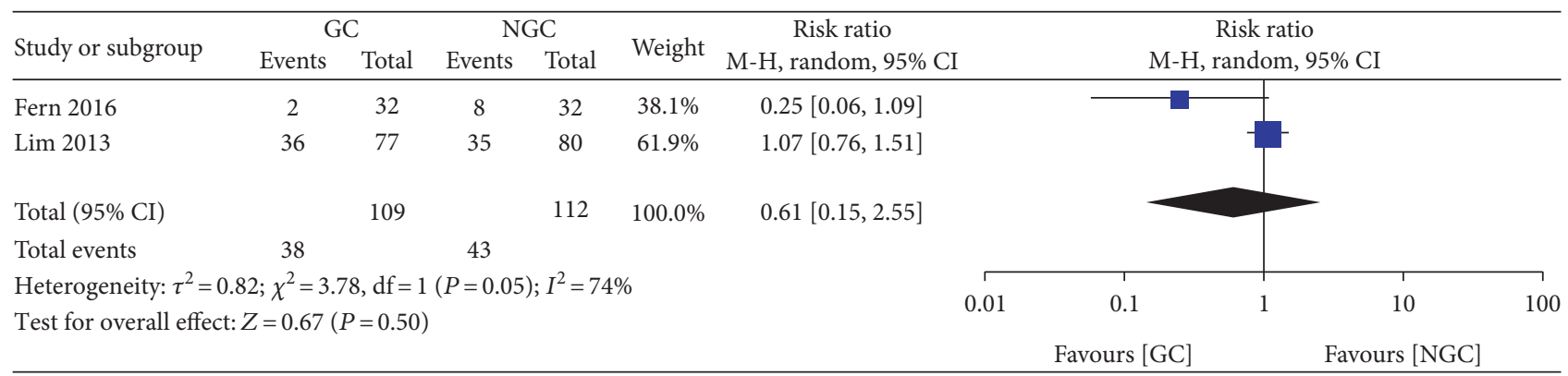

FIGURE 10: Forest plot for postoperative vomiting.

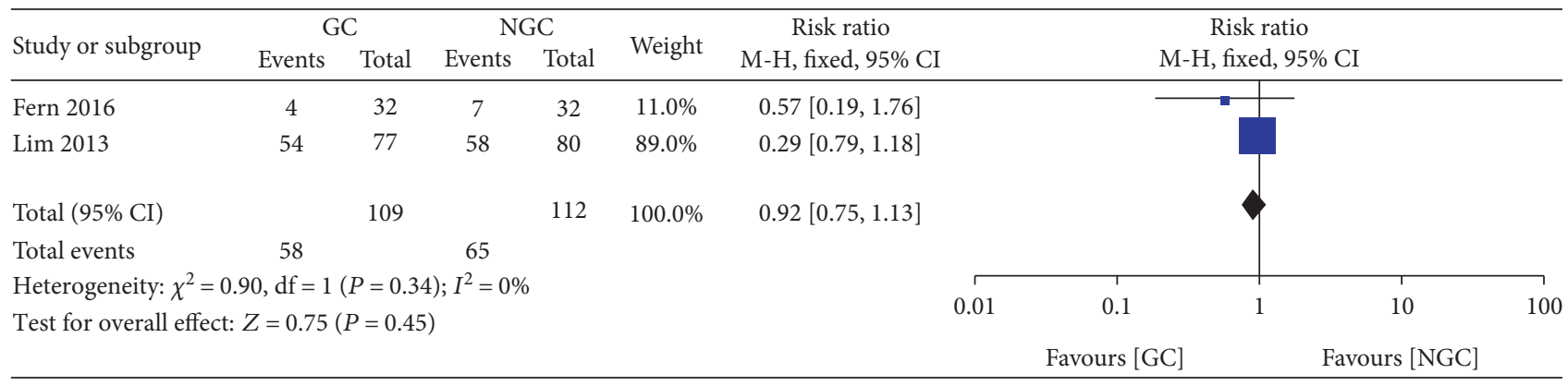

FIGURE 11: Forest plot for postoperative abdominal distention. 


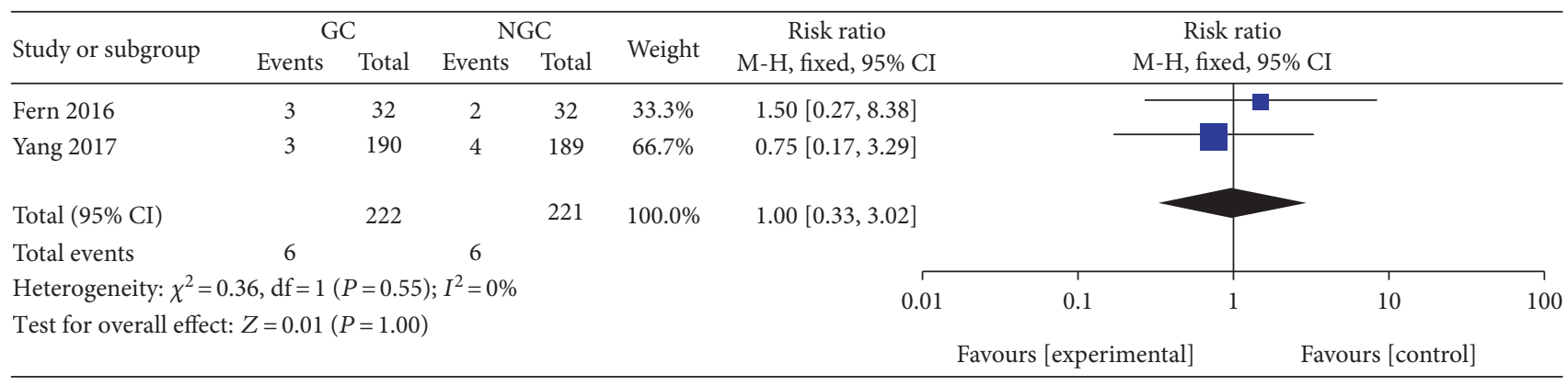

FIGURE 12: Forest plot for postoperative pneumonia.

\begin{tabular}{|c|c|c|c|c|c|c|c|c|c|c|c|c|c|}
\hline \multirow{3}{*}{$\begin{array}{l}\text { Study or subgroup } \\
\text { Asao } 2002\end{array}$} & \multicolumn{2}{|c|}{$\mathrm{GC}$} & \multicolumn{4}{|c|}{ NGC } & \multirow{3}{*}{$\begin{array}{c}\text { Weight } \\
2.2 \%\end{array}$} & \multirow{3}{*}{$\begin{array}{c}\text { Mean difference } \\
\text { IV, random, 95\% CI } \\
-1.00[-5.40,3.40]\end{array}$} & \multirow{2}{*}{\multicolumn{5}{|c|}{$\begin{array}{c}\text { Mean difference } \\
\text { IV, random, 95\% CI }\end{array}$}} \\
\hline & \multirow{2}{*}{$\frac{\text { Mean }}{13.5}$} & \multirow{2}{*}{$\frac{\mathrm{SD}}{3}$} & \multirow{2}{*}{$\begin{array}{c}\text { Total } \\
10\end{array}$} & \multirow{2}{*}{$\frac{\text { Mean }}{14.5}$} & \multirow{2}{*}{$\begin{array}{l}S \mathrm{SD} \\
6.1\end{array}$} & \multirow{2}{*}{$\begin{array}{c}\text { Total } \\
9\end{array}$} & & & & & & & \\
\hline & & & & & & & & & & & & & \\
\hline Fern 2016 & 7.5 & 5.1 & 32 & 9 & 5.7 & 32 & $4.9 \%$ & $-1.50[-4.15,1.15]$ & & & & & \\
\hline Forrester 2014 & 6.31 & 5.47 & 13 & 6.26 & 5.83 & 18 & $2.6 \%$ & $0.05[-3.96,4.06]$ & & & & & \\
\hline Heijkant 2015 & 9.5 & 4.9 & 58 & 14 & 14.5 & 62 & $2.8 \%$ & $-4.50[-8.32,-0.68]$ & $<$ & & & & \\
\hline Kobayashi 2015 & 19.8 & 12.6 & 21 & 16.4 & 16.9 & 22 & $0.6 \%$ & $3.40[-5.48,12.18]$ & & & & & \\
\hline Matros 2006 & 4.92 & 0.42 & 22 & 4.73 & 0.27 & 21 & $15.6 \%$ & $0.19[-0.02,0.40]$ & & & $=-$ & & \\
\hline McCormick 2005 & 4.8 & 1.3 & 53 & 5.3 & 1.95 & 35 & $13.5 \%$ & $-0.50[-1.23,0.23]$ & & & & & \\
\hline Quah 2006 & 9.4 & 2.5 & 19 & 11.1 & 7.3 & 19 & $3.3 \%$ & $-1.70[-5.17,1.77]$ & & & & & \\
\hline Schuster 2016 & 4.3 & 0.43 & 17 & 6.8 & 1.38 & 17 & $13.7 \%$ & $-2.50[-3.19,-1.81]$ & & & & & \\
\hline Topcu 2016 & 7.63 & 1.47 & 30 & 9.47 & 2.67 & 30 & $11.5 \%$ & $-1.84[-2.93,-0.75]$ & & & & & \\
\hline Yang 2017 & 10.5 & 2.5 & 190 & 10.9 & 2.4 & 189 & $14.7 \%$ & $-0.40[-0.89,0.09]$ & & & & & \\
\hline Zaghiyan 2013 & 4.7 & 0.7 & 54 & 4.5 & 19 & 60 & $14.6 \%$ & $0.20[-0.32,0.72]$ & & & & & \\
\hline Total $(95 \%$ CI $)$ & & & 519 & & & 514 & $100.0 \%$ & $-0.88[-1.59,-0.17]$ & & & & & \\
\hline $\begin{array}{l}\text { Heterogeneity: } \tau^{2}= \\
\text { Test for overall effec }\end{array}$ & $\begin{array}{l}83 ; \chi^{2}= \\
Z=2 .\end{array}$ & $\begin{array}{l}=75.34 \\
3(P=\end{array}$ & $\begin{array}{l}\mathrm{df}=1 \\
0.01)\end{array}$ & $(P<0.0$ & 0001) & $I^{2}=85$ & & & -4 & -2 & 0 & 2 & \\
\hline & & & & & & & & & & Favours [GC] & & Favours | & \\
\hline
\end{tabular}

FIGURE 13: Forest plot for the length postoperative hospital stay.

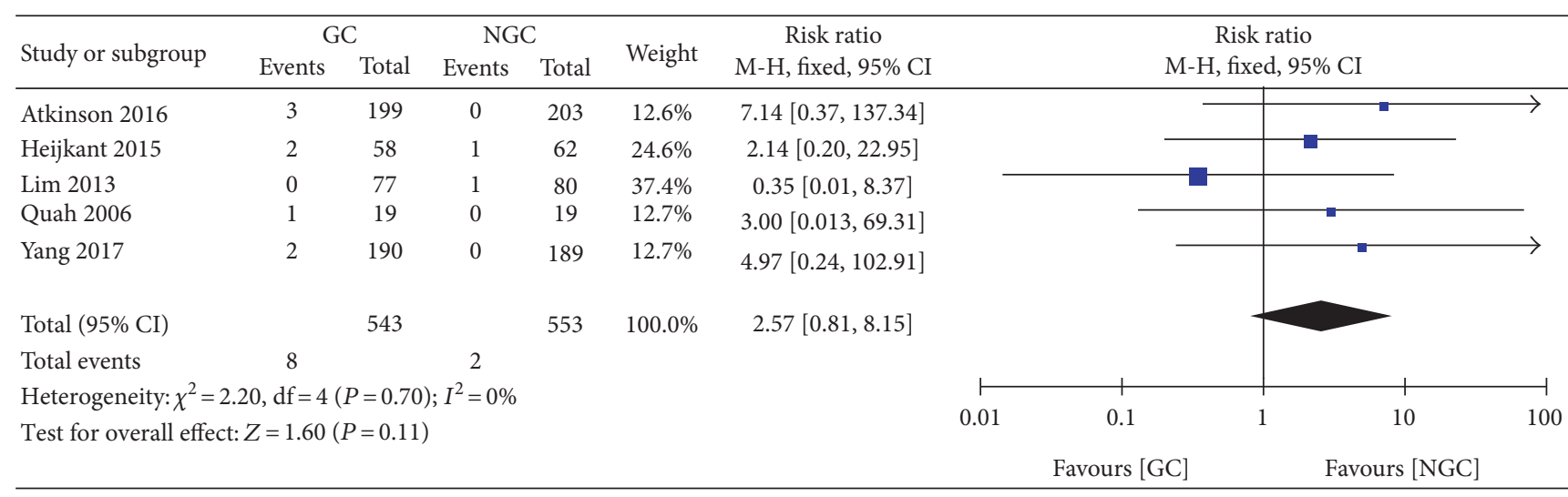

FIGURE 14: Forest plot for mortality.

(Figure 9), with a moderate statistical heterogeneity across the trials $\left(I^{2}=50 \%\right)$.

3.5.7. Postoperative Vomiting. There was no significant difference between the groups in the incidence of postoperative vomiting (RR $0.61 ; 95 \%$ CI 0.15 to $2.55 ; \quad P=0.50$ )
(Figure 10), with a clear statistical heterogeneity between trials $\left(I^{2}=74 \%\right)$.

3.5.8. Postoperative Abdominal Distension. There was no significant difference between the groups in the incidence of postoperative abdominal distension (RR 0.92; 95\% CI 0.75 
to $1.13 ; P=0.45)$ (Figure 11), with no statistical heterogeneity between trials $\left(P=0.34 ; I^{2}=0 \%\right)$.

3.5.9. Postoperative Pneumonia. No significant difference was observed between the gum chewing group and control group in the incidence of postoperative pneumonia (RR 1.00; 95\% CI 0.33 to $3.02 ; P=1.00$ ) (Figure 12), with no statistical heterogeneity between trials $\left(P=0.55 ; I^{2}=0 \%\right)$.

3.5.10. The Length of Postoperative Hospital Stay. Twelve studies reported the length of postoperative hospital stay after colorectal surgery under gum chewing intervention or standard nursing. The meta-analysis showed a statistically significant reduction in the length of postoperative hospital stay for the gum chewing group (WMD -0.88 ; $95 \% \mathrm{CI}-1.59$ to $-0.17 ; P=0.01$ ) (Figure 13 ), with a clear statistical heterogeneity across the studies $\left(I^{2}=85 \%\right)$.

3.5.11. Mortality. There was no significant difference between the groups in the incidence of mortality (RR $2.57 ; 95 \%$ CI 0.81 to $8.15 ; P=0.11$ ) (Figure 14 ), with no evidence of heterogeneity across the trials $\left(P=0.70 ; I^{2}=0 \%\right)$.

3.6. Intention to Treat Analysis. In the included studies, ten studies reported the individual withdrawal after randomization. In order to provide unbiased assessments of treatment efficacy, intention to treat analysis was conducted. The result showed that no obvious differences were found in postoperative nausea, vomiting, abdominal distention, and mortality between the gum chewing group and control group $(P>0.05)$, which was consistent with the aforementioned findings (Figure 15).

\section{Discussion}

The results of our meta-analysis have authenticated that chewing gum after colorectal cancer surgery is helpful for the recovery of the patient, which was associated with the reduction of the time to first passage of flatus, first bowel movement, start feeding, and the length of postoperative hospital stay. However, no difference was observed in the incidence of postoperative nausea, vomiting, abdominal distension, pneumonia, and mortality. In the included studies, most of them described the inclusion and exclusion criteria for disease, age, and informed consent, yet only one study [19] mentioned that patients were excluded if they had allergies to chewing gum, and just one research [17] presented the acceptability and compliance of chewing gum, which may result in a risk of selection bias. Additionally, ten studies reported the individual withdrawal after randomization. Remarkably, the main reasons for the patient's withdrawal from the study or not included in the analyses were that patients did not receive chewing gum and inability to chew gum. In order to provide unbiased assessments of treatment efficacy, intention to treat analysis was also conducted in our study. Similarly, we found that there were no obvious differences in postoperative nausea, vomiting, abdominal distention, and mortality between two arms. Complex and various influencing factors of long-term outcome and fewer data were incorporated regarding the outcome of postoperative complications that may account for the phenomenon. In our study, we only included the colorectal surgery but not the whole abdominal surgery (cesarean section, and others) with the following consideration. On the one hand, not all abdominal surgery actually relate to the gastrointestinal tract, and the process of intestinal function recovery may be different following different surgery. Chewing gum acts as sham feeding which, therefore, may promote the recovery of gastrointestinal function through various ways. On the other hand, colorectal surgery changes and affects the integrity of the intestinal tract. The recovery of intestinal function may mainly depend on the process of intestinal tract itself, and chewing gum may provide limited effects on reducing some complications, which just could explain our findings.

How gum chewing works remains unclear. The possible presupposition is that gum chewing mimics the mechanism of food intake, which may significantly stimulate motility in the stomach, duodenum, and rectum [33]; promote gastric, pancreatic, and duodenal secretion [34, 35]; and enhance the release of neuropeptides [33]. The proposed physiologic mechanism is that gum chewing activates the cephalic-vagal axis, which stimulates intestinal myoelectric activity to offset the activation of gastrointestinal opioid receptors [36]. In addition, gum chewing may also stimulate the saliva secretion, resulting in the production of nitrous oxide in sufficient quantities to combat the pathogens in the mouth and gut [37]. Furthermore, gum chewing may offer a better option to regulate a potential risk which is associated with early postoperative enteral or oral feeding.

Recently, a significant amount of research focused on the possible role of chewing gum in the intestinal function recovery, yet the evidence from RCTs remains inconclusive; thereupon, several meta-analyses of the efficacy of gum chewing were published. The meta-analysis by Su'a et al. [12] described the effects of chewing gum on postoperative ileus in adults, showing that chewing gum is beneficial to reducing the time to flatus and the time to bowel motion, but not the length of hospital stay or complications. It is noteworthy that the included patients in this meta-analysis receiving either colorectal surgery or cesarean sections resulted in a heterogeneous patient group. Another meta-analysis [7] of 10 RCTs suggested that sham feeding following colorectal surgery is safe, leading to the improvement in GI recovery, and is associated with the reduction in the length of hospital stay, which is in consistent with our results. Nevertheless, this study found that gum chewing conferred no advantage if patients are placed on a rapid postoperative feeding regime; while our study revealed that gum chewing might accelerate the time to first feeding, the difference may be explained by performing subgroup analysis on whether the rapid postoperative feeding was given to patients, which is just one of the limitations of our research that the corresponding analysis was not implemented. An earlier meta-analysis by Noble et al. [13] identifying nine eligible trials also showed that chewing sugarless gum following elective intestinal resection was associated with improved outcomes, but the efficacy on a reduced rate of clinical complications or reduced cost was not confirmed, which is also in line with our findings. Other 


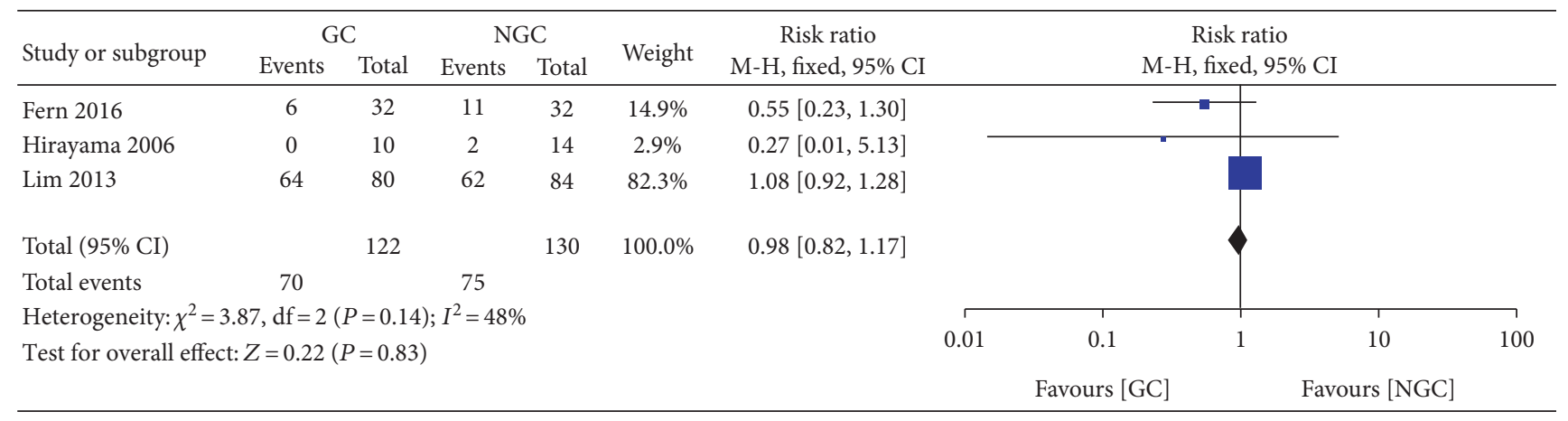

(a) Forest plot for postoperative nausea

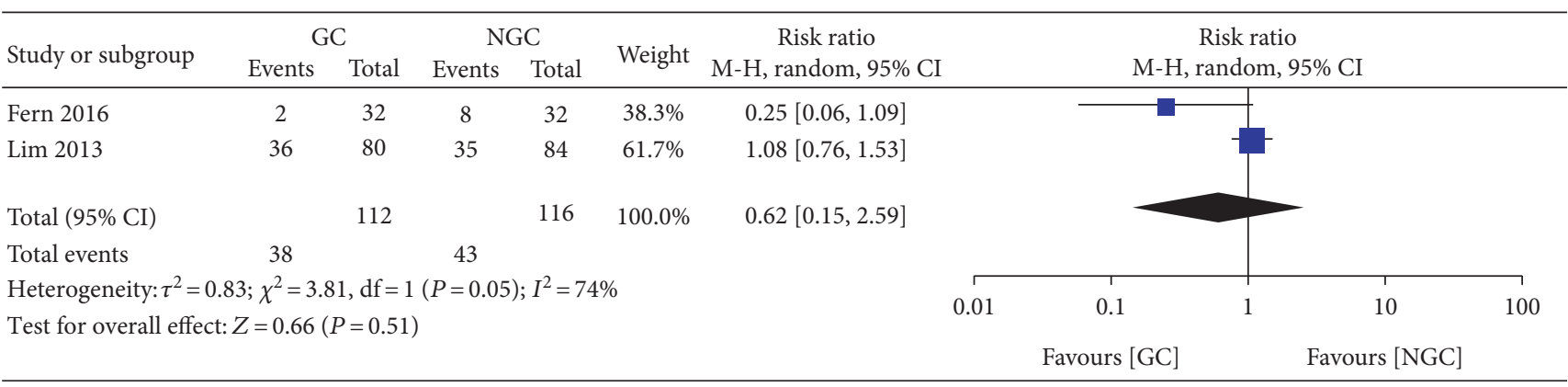

(b) Forest plot for postoperative vomiting

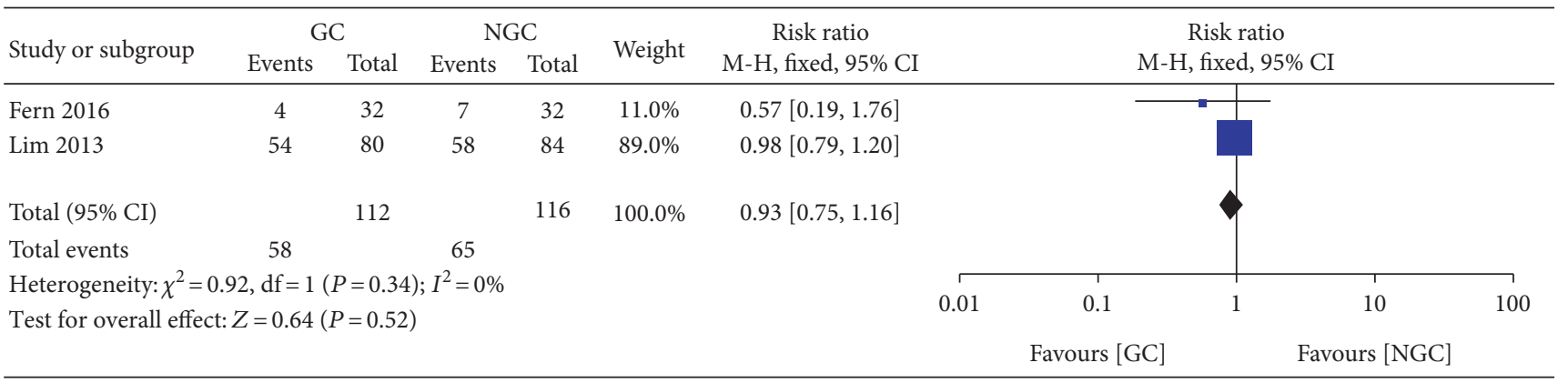

(c) Forest plot for postoperative abdominal distention

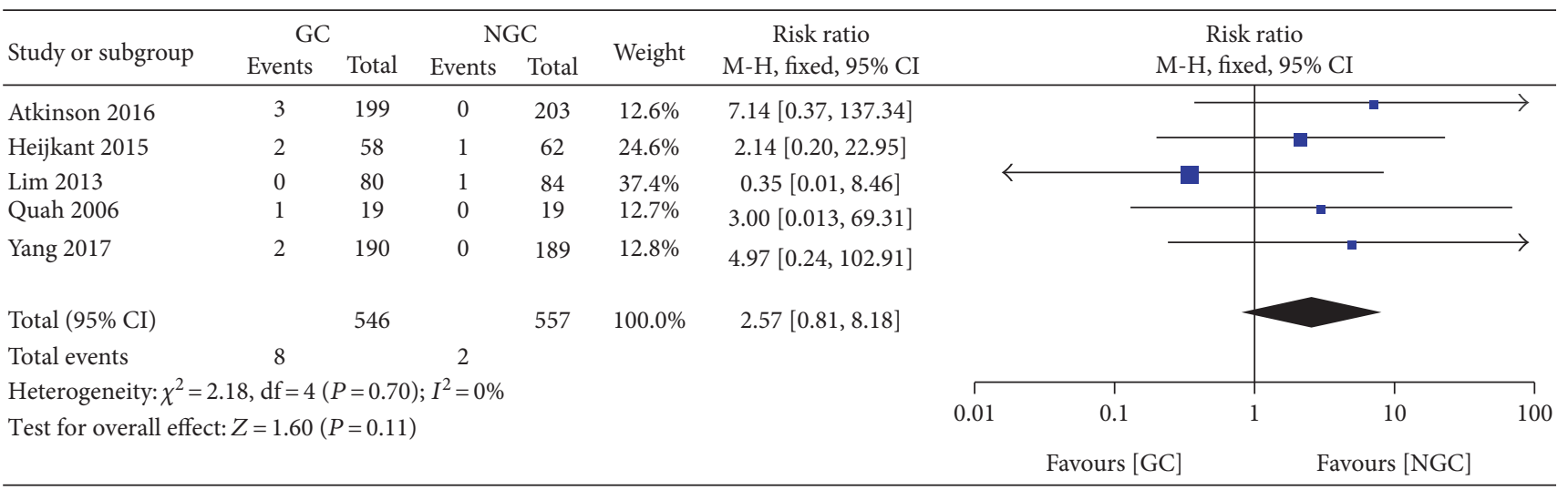

(d) Forest plot for mortality

Figure 15: Intention to treat analysis.

previous meta-analyses [38-44] that focused on the effects of gum chewing on the recovery of intestinal function after abdominal surgery (e.g., cesarean delivery) also confirmed the beneficial role of gum chewing after surgery. Currently, several RCTs with moderate or large sample size investigating the efficacy of chewing gum in patients undergoing colorectal resection have been published, however, which has not been included for synthesized analysis in the latest meta-analysis by Song et al. [45]. Consequently, we launch this updated meta-analysis to comprehensively 
evaluate the effect and safety of chewing gum for patients undergoing colorectal resection, which may have more advantages in reducing heterogeneity and publication bias.

Several limitations of this study should be addressed. Firstly, owing to the differences in patient population and surgery approaches involved, this meta-analysis is limited by the significant heterogeneity of the included studies. Additionally, limited included data subgroup analysis regarding different interventions (e.g., initiation and frequency) in gum chewing was not performed. Besides, there was a lack of blinding in most included studies which may have resulted in significant performance and detection bias. Although blinding participants may be difficult in this setting, blinding observers should be feasible and necessary which is conducive to increasing the reliability of the findings. Therefore, to improve the applicability of study results to individual patients and provide potentially feasible clinical practice guidelines for postoperative nursing, investigators should improve study design to ensure protocol adherence with minimal loss to selection bias risk and follow-up. Moreover, based on the distribution characteristics of prognostic features, how to explore potential causes and search for more powerful evidence still remain to be explored further.

\section{Conclusions}

This meta-analysis indicates that gum chewing in patients undergoing colorectal cancer surgery has a beneficial role in intestinal function recovery. However, the limited data suggests that it offers no benefit in reducing postoperative clinical complications such as nausea, vomiting, abdominal distension, pneumonia, and mortality. Prospective and blinded RCTs are warranted to further clarify the role of chewing gum in the postoperative care in colorectal cancer patients.

\section{Conflicts of Interest}

The authors declare that they have no conflict of interest.

\section{Authors' Contributions}

Binbin Mei and Wenting Wang are co-first authors. Feifei Cui and Zunjia Wen contributed equally to this work.

\section{References}

[1] C. J. van de Velde, C. Aristei, P. G. Boelens et al., "EURECCA colorectal: multidisciplinary mission statement on better care for patients with colon and rectal cancer in Europe," European Journal of Cancer, vol. 49, no. 13, pp. 2784-2790, 2013.

[2] E. J. Kuipers, W. M. Grady, D. Lieberman et al., "Colorectal cancer," Nature Reviews Disease Primers, vol. 1, p. 15065, 2015.

[3] A. Cimitan, T. Contardo, R. Molaro, and E. Morpurgo, "The role of laparoscopy in the treatment of anastomotic leaks after minimally invasive colorectal resections for cancer," Surgical Laparoscopy, Endoscopy \& Percutaneous Techniques, vol. 26, no. 4, pp. e80-e84, 2016.

[4] W. J. Tan, M. H. Chew, A. R. Dharmawan et al., "Critical appraisal of laparoscopic vs open rectal cancer surgery,"
World Journal of Gastrointestinal Surgery, vol. 8, no. 6, pp. 452-460, 2016.

[5] H. K. Andersen, S. J. Lewis, and S. Thomas, "Early enteral nutrition within $24 \mathrm{~h}$ of colorectal surgery versus later commencement of feeding for postoperative complications," The Cochrane Database of Systematic Reviews, vol. 18, no. 4, article CD004080, 2006.

[6] D. G. Watt, S. T. McSorley, P. G. Horgan, and M. M. DC, "Enhanced recovery after surgery: which components, if any, impact on the systemic inflammatory response following colorectal surgery?: a systematic review," Medicine, vol. 94, no. 36, article e1286, 2015.

[7] Y. M. Ho, S. R. Smith, P. Pockney, P. Lim, and J. Attia, "A meta-analysis on the effect of sham feeding following colectomy: should gum chewing be included in enhanced recovery after surgery protocols?," Diseases of the Colon and Rectum, vol. 57, no. 1, pp. 115-126, 2014.

[8] J. A. Lunding, L. M. Nordstrom, A. O. Haukelid, O. H. Gilja, A. Berstad, and T. Hausken, "Vagal activation by sham feeding improves gastric motility in functional dyspepsia," Neurogastroenterology \& Motility, vol. 20, no. 6, pp. 618-624, 2008.

[9] B. U. Su'a and A. G. Hill, "Perioperative use of chewing gum affects the inflammatory response and reduces postoperative ileus following major colorectal surgery," Evidence-Based Medicine, vol. 20, no. 5, pp. 185-186, 2015.

[10] P. Alfonsi, K. Slim, M. Chauvin et al., "Guidelines for enhanced recovery after elective colorectal surgery," Annales francaises d'anesthesie et de reanimation, vol. 33, no. 5, pp. 370-384, 2014.

[11] N. Jain and S. Mercer, "The chewing gum controversy-time for a consensus?," Journal of Clinical Anesthesia, vol. 27, no. 6, pp. 531-532, 2015.

[12] B. U. Su'a, T. T. Pollock, D. P. Lemanu, A. D. MacCormick, A. B. Connolly, and A. G. Hill, "Chewing gum and postoperative ileus in adults: a systematic literature review and meta-analysis," International Journal of Surgery, vol. 14, pp. 49-55, 2015.

[13] E. J. Noble, R. Harris, K. B. Hosie, S. Thomas, and S. J. Lewis, "Gum chewing reduces postoperative ileus? A systematic review and meta-analysis," International Journal of Surgery, vol. 7, no. 2, pp. 100-105, 2009.

[14] S. M. de Castro, J. W. van den Esschert, N. T. van Heek et al., "A systematic review of the efficacy of gum chewing for the amelioration of postoperative ileus," Digestive Surgery, vol. 25, no. 1, pp. 39-45, 2008.

[15] J. P. T. Higgins and S. Green, Cochrane Handbook for Systematic Reviews of Interventions Version 5.1.0 [updated March 2011], The Cochrane Collaboration, 2011.

[16] T. Asao, H. Kuwano, J. Nakamura, N. Morinaga, I. Hirayama, and M. Ide, "Gum chewing enhances early recovery from postoperative ileus after laparoscopic colectomy," Journal of the American College of Surgeons, vol. 195, no. 1, pp. 30-32, 2002.

[17] C. Atkinson, C. M. Penfold, A. R. Ness et al., "Randomized clinical trial of postoperative chewing gum versus standard care after colorectal resection," The British Journal of Surgery, vol. 103, no. 8, pp. 962-970, 2016.

[18] O. Vergara-Fernandez, A. P. Gonzalez-Vargas, J. C. Castellanos-Juarez, N. Salgado-Nesme, and E. Sanchez-Garcia Ramos, "Usefulness of gum chewing to decrease postoperative ileus in colorectal surgery with primary anastomosis: a randomized controlled trial," Revista de Investigacion Clinica; 
organo del Hospital de Enfermedades de la Nutricion, vol. 68, no. 6, pp. 314-318, 2016.

[19] D. A. Forrester, J. Doyle-Munoz, T. McTigue, S. D'Andrea, and A. Natale-Ryan, "The efficacy of gum chewing in reducing postoperative ileus: a multisite randomized controlled trial," Journal of Wound, Ostomy \& Continence Nursing, vol. 41, no. 3, pp. 227-232, 2014.

[20] T. C. van den Heijkant, L. M. Costes, D. G. van der Lee et al., "Randomized clinical trial of the effect of gum chewing on postoperative ileus and inflammation in colorectal surgery," The British Journal of Surgery, vol. 102, no. 3, pp. 202-211, 2015.

[21] I. Hirayama, M. Suzuki, M. Ide, T. Asao, and H. Kuwano, "Gum-chewing stimulates bowel motility after surgery for colorectal cancer," Hepato-Gastroenterology, vol. 53, no. 68, pp. 206-208, 2006.

[22] D. Y. Hwang, H. Y. Kim, J. H. Kim et al., "Effect of gum chewing on the recovery from laparoscopic colorectal cancer surgery," Annals of Coloproctology, vol. 29, no. 6, pp. 248-251, 2013.

[23] T. Kobayashi, T. Masaki, K. Kogawa, H. Matsuoka, and M. Sugiyama, "Efficacy of gum chewing on bowel movement after open colectomy for left-sided colorectal cancer: a randomized clinical trial," Diseases of the Colon and Rectum, vol. 58, no. 11, pp. 1058-1063, 2015.

[24] P. Lim, O. J. Morris, G. Nolan, S. Moore, B. Draganic, and S. R. Smith, "Sham feeding with chewing gum after elective colorectal resectional surgery: a randomized clinical trial," Annals of Surgery, vol. 257, no. 6, pp. 1016-1024, 2013.

[25] E. Matros, F. Rocha, M. Zinner et al., "Does gum chewing ameliorate postoperative ileus? Results of a prospective, randomized, placebo-controlled trial," Journal of the American College of Surgeons, vol. 202, no. 5, pp. 773-778, 2006.

[26] J. T. McCormick, R. Garvin, P. Caushaj et al., "The effects of gum-chewing on bowel function and hospital stay after laparoscopic vs open colectomy: a multi-institution prospective randomized trial," Journal of the American College of Surgeons, vol. 201, no. 3, Supplement 3, pp. S66-S67, 2005.

[27] H. M. Quah, A. Samad, A. J. Neathey, D. J. Hay, and A. Maw, "Does gum chewing reduce postoperative ileus following open colectomy for left-sided colon and rectal cancer? A prospective randomized controlled trial," Colorectal Disease, vol. 8, no. 1, pp. 64-70, 2006.

[28] R. Schuster, N. Grewal, G. C. Greaney, and K. Waxman, "Gum chewing reduces ileus after elective open sigmoid colectomy," Archives of Surgery, vol. 141, no. 2, pp. 174-176, 2006.

[29] N. F. Shum, H. K. Choi, J. C. Mak, D. C. Foo, W. C. Li, and W. L. Law, "Randomized clinical trial of chewing gum after laparoscopic colorectal resection," The British Journal of Surgery, vol. 103, no. 11, pp. 1447-1452, 2016.

[30] S. Y. Topcu and S. D. Oztekin, "Effect of gum chewing on reducing postoperative ileus and recovery after colorectal surgery: a randomised controlled trial," Complementary Therapies in Clinical Practice, vol. 23, pp. 21-25, 2016.

[31] Y. Yang, H. Q. Zuo, Z. Li et al., "Comparison of efficacy of simo decoction and acupuncture or chewing gum alone on postoperative ileus in colorectal cancer resection: a randomized trial," Scientific Reports, vol. 7, article 37826, 2017.

[32] K. Zaghiyan, S. Felder, G. Ovsepyan et al., "A prospective randomized controlled trial of sugared chewing gum on gastrointestinal recovery after major colorectal surgery in patients managed with early enteral feeding," Diseases of the Colon and Rectum, vol. 56, no. 3, pp. 328-335, 2013.
[33] E. E. Soffer and T. E. Adrian, "Effect of meal composition and sham feeding on duodenojejunal motility in humans," Digestive Diseases and Sciences, vol. 37, no. 7, pp. 1009-1014, 1992.

[34] S. J. Konturek and P. Thor, "Relation between duodenal alkaline secretion and motility in fasted and sham-fed dogs," The American Journal of Physiology, vol. 251, 5 Part 1, pp. G591-G596, 1986.

[35] J. M. Jepsen, E. Skoubo-Kristensen, and L. Elsborg, "Rectosigmoid motility response to sham feeding in irritable bowel syndrome. Evidence of a cephalic phase," Scandinavian Journal of Gastroenterology, vol. 24, no. 1, pp. 53-56, 1989.

[36] J. Fanning and F. A. Valea, "Perioperative bowel management for gynecologic surgery," American Journal of Obstetrics and Gynecology, vol. 205, no. 4, pp. 309-314, 2011.

[37] C. Duncan, H. Dougall, P. Johnston et al., "Chemical generation of nitric oxide in the mouth from the enterosalivary circulation of dietary nitrate," Nature Medicine, vol. 1, no. 6, pp. 546-551, 1995.

[38] H. P. Huang and M. He, "Usefulness of chewing gum for recovering intestinal function after cesarean delivery: a systematic review and meta-analysis of randomized controlled trials," Taiwanese Journal of Obstetrics \& Gynecology, vol. 54, no. 2, pp. 116-121, 2015.

[39] Y. P. Zhu, W. J. Wang, S. L. Zhang, B. Dai, and D. W. Ye, "Effects of gum chewing on postoperative bowel motility after caesarean section: a meta-analysis of randomised controlled trials," BJOG : An International Journal of Obstetrics \& Gynaecology, vol. 121, no. 7, pp. 787-792, 2014.

[40] H. Hochner, S. M. Tenfelde, W. Abu Ahmad, and M. Liebergall-Wischnitzer, "Gum chewing and gastrointestinal function following caesarean delivery: a systematic review and meta-analysis," Journal of Clinical Nursing, vol. 24, no. 13-14, pp. 1795-1804, 2015.

[41] Z. Wen, M. Shen, C. Wu, J. Ding, and B. Mei, "Chewing gum for intestinal function recovery after caesarean section: a systematic review and meta-analysis," BMC Pregnancy and Childbirth, vol. 17, no. 1, p. 105, 2017.

[42] S. Li, Y. Liu, Q. Peng, L. Xie, J. Wang, and X. Qin, "Chewing gum reduces postoperative ileus following abdominal surgery: a meta-analysis of 17 randomized controlled trials," Journal of Gastroenterology and Hepatology, vol. 28, no. 7, pp. 11221132, 2013.

[43] W. Vasquez, A. V. Hernandez, and J. L. Garcia-Sabrido, "Is gum chewing useful for ileus after elective colorectal surgery? A systematic review and meta-analysis of randomized clinical trials," Journal of Gastrointestinal Surgery, vol. 13, no. 4, pp. 649-656, 2009.

[44] E. Sahin and F. Terzioglu, "The effect of gum chewing, early oral hydration, and early mobilization on intestinal motility after cesarean birth," Worldviews on Evidence-Based Nursing, vol. 12, no. 6, pp. 380-388, 2015.

[45] G. M. Song, Y. H. Deng, Y. H. Jin, J. G. Zhou, and X. Tian, "Meta-analysis comparing chewing gum versus standard postoperative care after colorectal resection," Oncotarget, vol. 7, no. 43, pp. 70066-70079, 2016. 


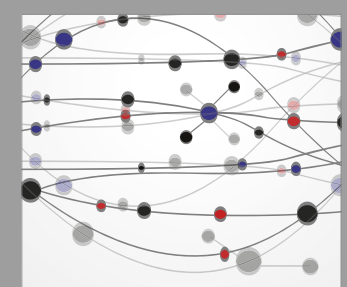

The Scientific World Journal
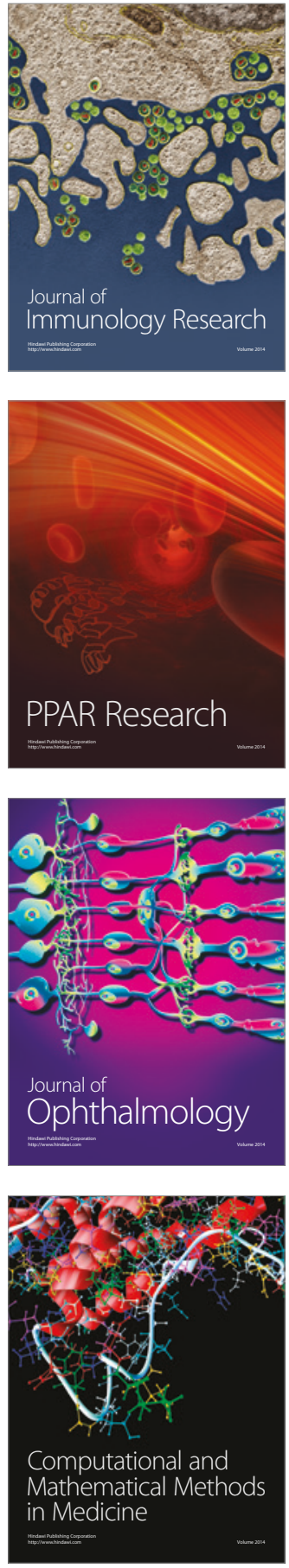

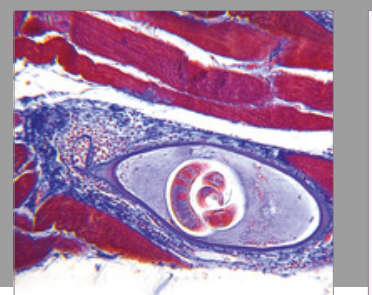

Gastroenterology Research and Practice
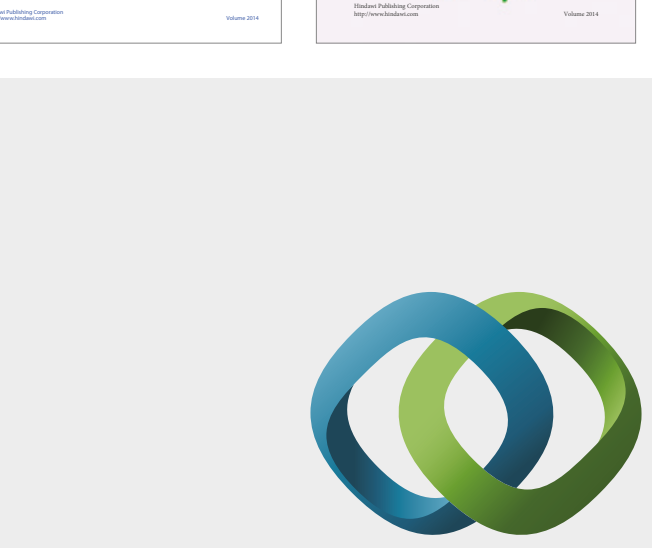

\section{Hindawi}

Submit your manuscripts at

https://www.hindawi.com
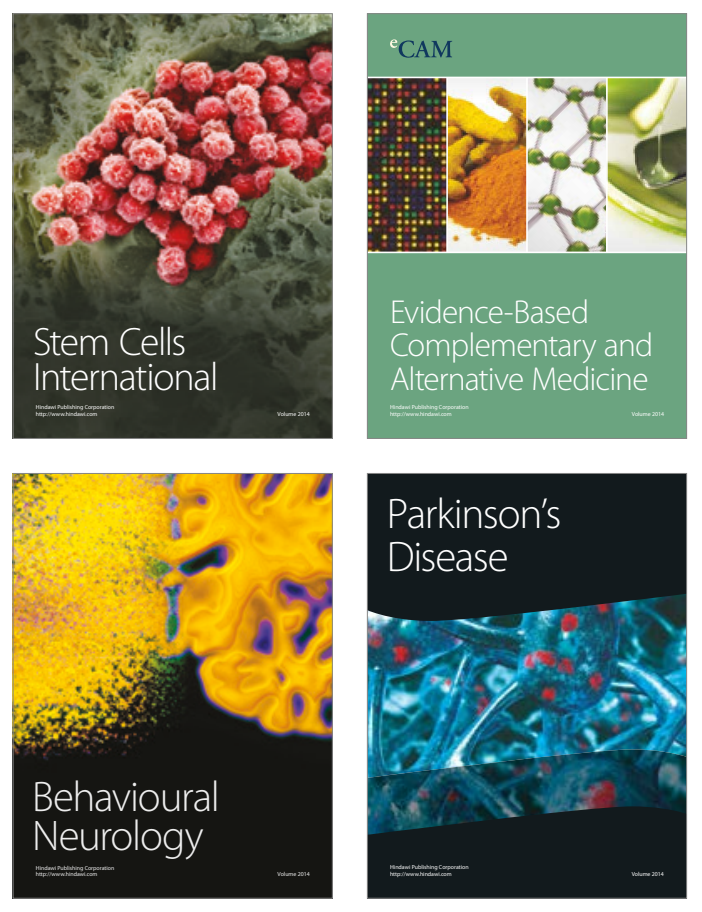
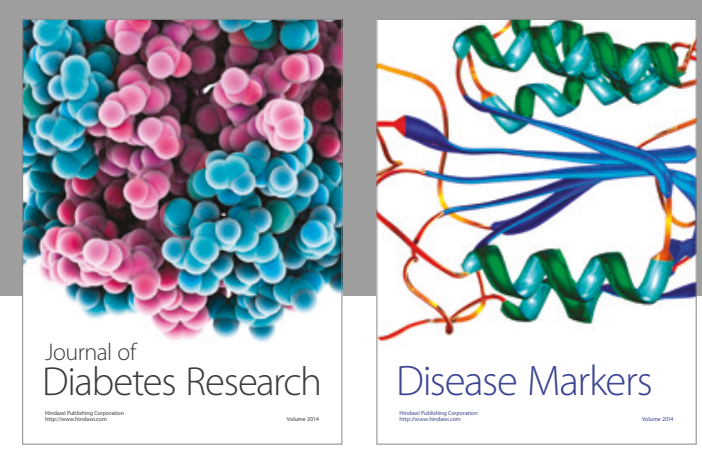

Disease Markers
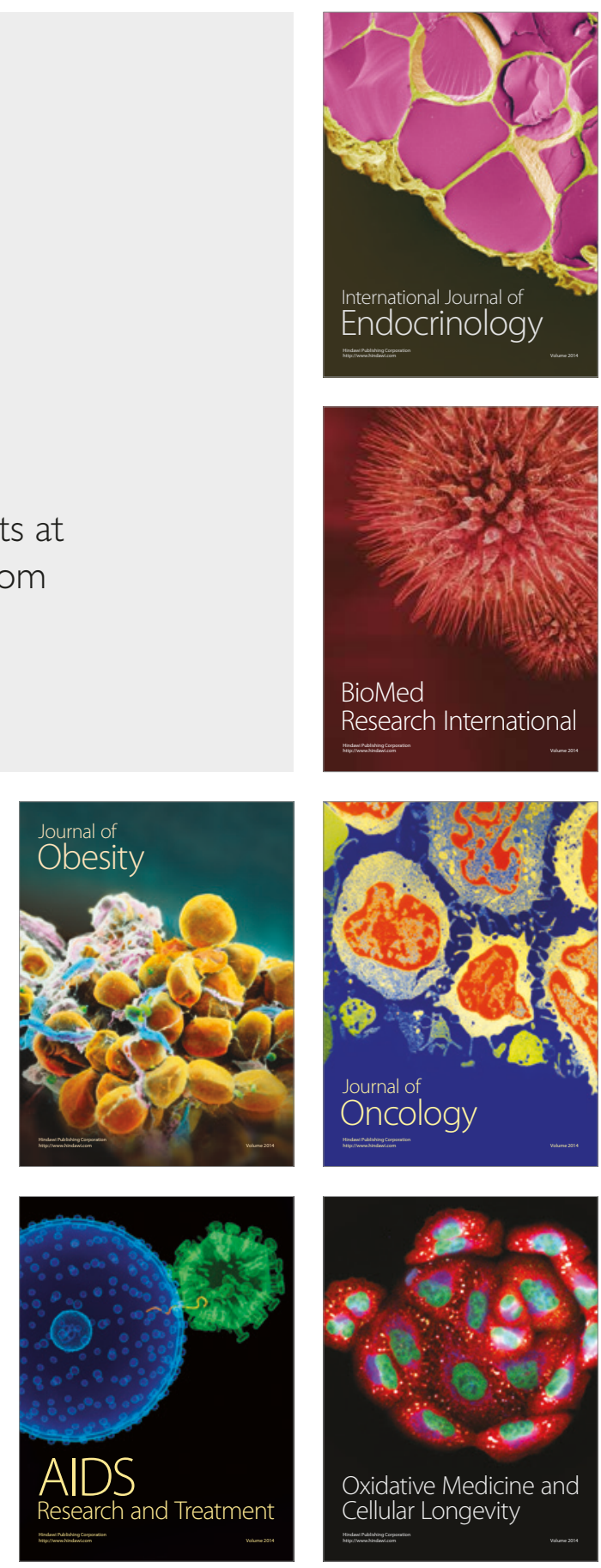